\title{
Thermodynamic Properties of Yttrium Based Cuprate Due to Electron-Cooper Pair Interaction Using BVT
}

\author{
Abel Wamalwa Mukubwa, Jared Oloo Odhiambo, John Wanjala Makokha \\ Department of Science, Technology and Engineering, Kibabii University, Bungoma, Kenya \\ Email: abelmuwa@gmail.com
}

How to cite this paper: Mukubwa, A.W., Odhiambo, J.O. and Makokha, J.W. (2018) Thermodynamic Properties of Yttrium Based Cuprate Due to Electron-Cooper Pair Interaction Using BVT. Open Access Library Journal, 5: e4880.

https://doi.org/10.4236/oalib.1104880

Received: September 5, 2018

Accepted: October 12, 2018

Published: October 15, 2018

Copyright $\odot 2018$ by authors and Open Access Library Inc.

This work is licensed under the Creative Commons Attribution International License (CC BY 4.0).

http://creativecommons.org/licenses/by/4.0/ (c) () Open Access

\begin{abstract}
Since the discovery of high temperature superconductivity (HTSC), there have been varied results concerning the high pairing energy in the unconventional superconductivity. In an attempt to unearth the mechanism behind the high pairing energy, we study a model in which an excited electron interacts with a Cooper pair. The interaction was studied earlier on using second quantization which depended on a wave function. We now use Bogoliubov-Valatini transformation (BVT) that is independent of the wave function to study the thermodynamic properties due to the interaction between an electron and a Cooper pair in a Yttrium based cuprate. The interaction energy increases with temperature and at $T=93 \mathrm{~K}$; we record the interaction energy of $2.173 \mathrm{meV}$ and the specific heat of $4.669 \mathrm{~J} \cdot \mathrm{mol}^{-1} \cdot \mathrm{K}^{-1}$. The entropy of this system is found to be $5.11 \mathrm{~mJ} \cdot \mathrm{g}^{-1} \cdot \mathrm{K}^{-1}$. The Sommerfeld's coefficient is maximum at $T=\frac{2}{3} T_{C}$ where $\gamma=62.4 \mathrm{~mJ} \cdot \mathrm{mol}^{-1} \cdot \mathrm{K}^{-2}$. It has also been observed, for the first time, that the thermodynamic quantities of YBCO123 are constant when temperature varies between $0 \mathrm{~K}$ and $18 \mathrm{~K}$. Above $18 \mathrm{~K}$, the thermodynamic quantities rise sharply. This observation has been attributed to the pairing of electrons in the singlet-s and singlet-p states.
\end{abstract}

\section{Subject Areas}

Chemical Engineering \& Technology

\section{Keywords}

Energy Gap, Specific Heat, Entropy

\section{Introduction}

The microscopic theory of superconductivity, based on s-wave pairing, has been 
used to explain conventional superconductivity [1]. However, the theory does not hold in the case of unconventional superconductivity [2]. The pairing mechanism in high temperature superconductors below the critical temperature remains unclear despite the enormous research carried out on HTSC [3]. There is need to develop consistence and consensus between the various theoretical approaches towards explaining the mechanism of HTSC. The energy gap plays a central role in explaining superconductivity, both conventional and unconventional. The energy gap in high temperature superconductor is non-uniform along the Fermi surface, characteristic of all cuprates [4].

$\mathrm{YBa}_{2} \mathrm{Cu}_{3} \mathrm{O}_{7}$, with a $T_{C}$ of $93 \mathrm{~K}$, was discovered a year later after the landmark discovery of a lanthanum-based cuprate [5] [6]. Later on, Bismuth and Lanthanum based cuprates were discovered [7] [8] [9] [10]. Sulfur hydride, under extremely high pressure of about $200 \mathrm{GPa}$ was found to undergo superconducting transition at a $T_{C}$ of $203 \mathrm{~K}$, the highest experimental $T_{C}$ that has ever been achieved in the history of superconductivity [11]. High temperature superconductors have a wide range of applications ranging from the maglev trains, Superconducting Quantum Interface Devices (SQUIDs) up to Magnetic Resonance Imaging (MRI). Understanding the pairing mechanism in HTSC superconductors will enhance theoretical predictions, with precision, of the $T_{C}$ 's as well as other thermodynamic properties of cuprates. This will open avenues for discoveries towards room-temperature superconductivity (RTS). Although many different HTSC cuprates have been discovered, theoretical as well as experimental studies are still conflicting with the observed properties [12] [13] [14]. Experimental studies have shown evidence of d-wave pairing in YBCO [2]. The Cooper pair in a high temperature superconductor has an angular momentum greater than zero, which implies the presence of nodes [15]. Electron-phonon interactions and spin fluctuations are central to the mechanism of superconductivity [16]. Spin fluctuations have sufficient strength to mediate the pairing interactions in high-temperature superconductivity except that the estimates of the strengths of this interaction differ widely [17]. Superconductivity majorly results from the formation of Cooper pairs, at the Fermi surface, at the critical temperature. However not all electrons at the Fermi surface form Cooper pairs, giving rise to a phenomenon in which Cooper pairs interact with electrons [3]. The interaction occurs between the copper oxide planes of the cuprate. When second quantization was applied on this model, the resulting entropy and specific heat of YBCO123 showed close proximity to the results from previously done experimental and theoretical work [14] [18] [19] [20]. However, the energy determined by the same method, varied from the previous experimental result [3] [21] [22]. This discrepancy might have been due to the method of second quantization used. In Bogoliubov-Valatini transformation (BVT), a Hamiltonian of interaction between the electron and the Cooper pairs is formed based on the kinetic energies of both the electron and the Cooper pair, the positive potential and the negative Coulombic potential. Row reducing the Hamiltonian leads to 
energy of the system at condensate state. The entropy and specific heat of the system have been deduced from the expression of the system's energy.

\section{Theoretical Formulation}

The Hamiltonian, $H_{0}$, of interaction between a Cooper pair in state $k$ and electron in state $q$ for unperturbed system is

$$
H_{0}=\sum_{q} \epsilon_{q} a_{q}^{\dagger} a_{q}+\sum_{k} \epsilon_{k} b_{k}^{\dagger} b_{k}
$$

where $a_{q}^{\dagger}\left(a_{q}\right)$ is the creation (annihilation) operator for an electron in state $q$, $b_{k}^{\dagger}=a_{k}^{\dagger} a_{-k}^{\dagger} \quad\left(b_{k}=a_{-k} a_{k}\right)$ is the creation (annihilation) operator for the Cooper pair in state $k, a_{q}^{\dagger} a_{q}$ and $b_{k}^{\dagger} b_{k}$ are the number operators for an electron and a Cooper pair respectively; $\epsilon_{q}=\hbar k_{e}^{2} / 2 m_{e}$ is the kinetic energy for electron and $\epsilon_{k}=\hbar k_{e}^{2} / 2 m_{c}$ is the kinetic energy for the Cooper pair.

The Hamiltonian for the perturbation, $H_{P}$, is given by

$$
H_{P}=\sum_{k, q} V_{k, q} a_{q}^{\dagger} a_{q}\left(b_{k}^{\dagger}-b_{k}\right)-\sum_{q, k} U_{k} a_{q}^{\dagger} a_{q} b_{k}^{\dagger} b_{k}
$$

where $V_{k, q}\left(U_{k, q}\right)$ is the interaction potential between electron and the Cooper pair.

Equations (1) and (2), when combined, give the Hamiltonian for a perturbed system as

$$
H=\sum_{q} \epsilon_{q} a_{q}^{\dagger} a_{q}+\sum_{k} \epsilon_{k} b_{k}^{\dagger} b_{k}+\sum_{k, q} V_{k, q} a_{q}^{\dagger} a_{q}\left(b_{k}^{\dagger}-b_{k}\right)-\sum_{q, k} U_{k} a_{q}^{\dagger} a_{q} b_{k}^{\dagger} b_{k}
$$

Equation (3) is then written in terms of Bogoliubov-Valatini operators, $\gamma$, using the relations $a_{k}=u_{q} \gamma_{q}+v_{q} \gamma_{-q}^{+}, \quad a_{-k}=u_{k} \gamma_{-k}-v_{k} \gamma_{k}^{+}, a_{k}^{\dagger}=u_{q} \gamma_{q}^{+}+v_{q} \gamma_{-q}$ and $a_{-k}^{\dagger}=u_{k} \gamma_{-k}^{+}-v_{k} \gamma_{k}$. In this case, $\left|u_{k}\right|^{2}$ is the probability that the pair state $\{\boldsymbol{k},-\boldsymbol{k}\}$ within a certain interval around the Fermi level is unoccupied and $\left|v_{k}\right|^{2}$ is the probability that the pair state $\{\boldsymbol{k},-\boldsymbol{k}\}$ within a certain interval around the Fermi level is occupied ( $\boldsymbol{k}$ is the wave vector). Thus, Equation (4) gives the total Hamiltonian of the system.

$$
\begin{aligned}
H= & \sum_{q} \epsilon_{q}\left\{u_{q}^{2} m_{q}+v_{q}^{2}\left(1-m_{-q}\right)+u_{q} v_{q}\left(\gamma_{q}^{+} \gamma_{-q}^{+}+\gamma_{-q} \gamma_{q}\right)\right\} \\
& +\sum_{k} \epsilon_{k}\left\{u_{k}^{4} m_{-k} m_{k}-u_{k}^{2} v_{k}^{2} m_{k}\left(1-m_{-k}\right)+u_{k}^{2} v_{k}^{2}\left(1-m_{k}\right) m_{k}\right. \\
& +u_{k}^{2} v_{k}^{2}\left(1-m_{-k}\right) m_{-k}-u_{k}^{2} v_{k}^{2}\left(1-m_{-k}\right) m_{k}+v_{k}^{4}\left(1-m_{-k}\right)\left(1-m_{k}\right) \\
& \left.+\left\{u_{k}^{3} v_{k}\left(m_{-k}+m_{k}\right)+v_{k}^{3} u_{k}\left(2-m_{k}-m_{-k}\right)\right\}\left(\gamma_{k}^{+} \gamma_{-k}^{+}+\gamma_{-k} \gamma_{k}\right)\right\} \\
& +\sum_{k, q} V_{k, q}\left\{\left\{u_{q}^{2} m_{q}+v_{q}^{2}\left(1-m_{-q}\right)\right\}\left(\gamma_{k}^{+} \gamma_{-k}^{+}-\gamma_{-k} \gamma_{k}\right)\right\} \\
& -\sum_{k, q} U_{k}\left\{u_{k}^{4} u_{q}^{2} m_{-k} m_{k} m_{q}+u_{k}^{4} v_{q}^{2} m_{k}\left(1-m_{-q}\right) m_{-k}\right. \\
& +u_{q}^{2} v_{k}^{4}\left(1-m_{k}\right)\left(1-m_{-k}\right) m_{q}+v_{q}^{2} v_{k}^{4}\left(1-m_{-k}\right)\left(1-m_{-q}\right)\left(1-m_{k}\right) \\
& +u_{q}^{2} u_{k}^{2} v_{k}^{2} m_{q}\left[m_{k}\left(1-m_{k}\right)-2 m_{k}\left(1-m_{-k}\right)+m_{-k}\left(1-m_{-k}\right)\right] \\
& +u_{k}^{2} v_{k}^{2} v_{q}^{2}\left(1-m_{-q}\right)\left[\left(1-m_{k}\right) m_{k}-2\left(1-m_{-k}\right) m_{k}+\left(1-m_{-k}\right) m_{-k}\right]
\end{aligned}
$$




$$
\begin{aligned}
& +\left[u_{k}^{3} u_{q}^{2} v_{k}\left(m_{-k}+m_{k}\right) m_{q}+u_{k}^{3} v_{q}^{2} v_{k}\left(m_{-k}+m_{k}\right)\left(1-m_{-q}\right)\right. \\
& \left.+v_{k}^{3} u_{q}^{2} u_{k}\left(2-m_{k}-m_{-k}\right) m_{q}+v_{k}^{3} v_{q}^{2} u_{k}\left(1-m_{-q}\right)\left(2-m_{k}-m_{-k}\right)\right]\left(\gamma_{k}^{+} \gamma_{-k}^{+}+\gamma_{-k} \gamma_{k}\right) \\
& +\left[u_{k}^{2} v_{k}^{2} u_{q} v_{q}\left(1-m_{-k}\right) m_{-k}-2 u_{k}^{2} v_{k}^{2} u_{q} v_{q} m_{k}\left(1-m_{-k}\right)+u_{k}^{2} v_{k}^{2} u_{q} v_{q}\left(1-m_{k}\right) m_{k}\right. \\
& \left.\left.+u_{k}^{4} u_{q} v_{q} m_{-k} m_{k}+v_{k}^{4} u_{q} v_{q}\left(1-m_{-k}\right)\left(1-m_{k}\right)\right]\left(\gamma_{q}^{+} \gamma_{-q}^{+}+\gamma_{-q} \gamma_{q}\right)\right\}+4 \mathrm{OT}
\end{aligned}
$$

where $4 \mathrm{OT}$ are the fourth order terms.

\subsection{Energy of the System}

The diagonal part of the effective Hamiltonian represents the energy of the system at equilibrium. Therefore, at equilibrium the energy of the system is given by Equation (5).

$$
\begin{aligned}
E_{k}= & \sum_{q} \epsilon_{q}\left\{u_{q}^{2} m_{q}+v_{q}^{2}\left(1-m_{-q}\right)\right\}+\sum_{k} \epsilon_{k}\left\{u_{k}^{4} m_{-k} m_{k}-u_{k}^{2} v_{k}^{2} m_{k}\left(1-m_{-k}\right)\right. \\
& +u_{k}^{2} v_{k}^{2}\left(1-m_{k}\right) m_{k}+u_{k}^{2} v_{k}^{2}\left(1-m_{-k}\right) m_{-k}-u_{k}^{2} v_{k}^{2}\left(1-m_{-k}\right) m_{k} \\
& \left.+v_{k}^{4}\left(1-m_{-k}\right)\left(1-m_{k}\right)\right\}-\sum_{k, q} U_{k, q}\left\{u_{k}^{4} u_{q}^{2} m_{-k} m_{k} m_{q}+u_{k}^{4} v_{q}^{2} m_{k}\left(1-m_{-q}\right) m_{-k}\right. \\
& +u_{q}^{2} v_{k}^{4}\left(1-m_{k}\right)\left(1-m_{-k}\right) m_{q}+v_{q}^{2} v_{k}^{4}\left(1-m_{-k}\right)\left(1-m_{-q}\right)\left(1-m_{k}\right) \\
& +u_{q}^{2} u_{k}^{2} v_{k}^{2} m_{q}\left[m_{k}\left(1-m_{k}\right)-2 m_{k}\left(1-m_{-k}\right)+m_{-k}\left(1-m_{-k}\right)\right] \\
& \left.+u_{k}^{2} v_{k}^{2} v_{q}^{2}\left(1-m_{-q}\right)\left[\left(1-m_{k}\right) m_{k}-2\left(1-m_{-k}\right) m_{k}+\left(1-m_{-k}\right) m_{-k}\right]\right\}
\end{aligned}
$$

At equilibrium, the quasi-particles represented by the operators $\gamma$ are very few or do not exist and therefore, $m_{k}=m_{-k}=0$ and $m_{q}=m_{-q}=0$. Thus, Equation (5) becomes

$$
E_{k}=\sum_{q} \epsilon_{q} v_{q}^{2}+\epsilon_{k} v_{k}^{4}-v_{k}^{4} \sum_{q} U_{k, q} v_{q}^{2}
$$

For the electron to interact with the Cooper pair, they must be in the same state i.e. at the time of interaction we only consider the state $k$ of the electron and neglect all the other states in $q$ and therefore Equation (7) becomes,

$$
E_{k}=\epsilon_{q} v_{k}^{2}+\epsilon_{k} v_{k}^{4}-v_{k}^{4} U_{k, k} v_{k}^{2}
$$

For the three-electron interaction to take place, the cooper pair and the electron involved in the interaction must be present. Thus, $v_{k}=1$ and $u_{k}=0$,

$$
E_{k}=\epsilon_{q}+\epsilon_{k}-U_{k, k}
$$

To introduce temperature dependence, the energy $E_{k}$ is multiplied by the thermal activation factor $\exp \left(\frac{-E_{k}}{K_{B} T}\right)$ where $E_{k}$ is energy of the system and $K_{B}$ is the Boltzmann constant. This produces a temperature dependent energy $E_{T}$ given as

$$
E_{T}=E_{k} \mathrm{e}^{\left(\frac{-E_{k}}{K_{B} T}\right)}
$$

The sum of kinetic energies $\left(\epsilon_{q}+\epsilon_{k}\right)$ and $U_{k, k}$ for Y123 have been determined as $10.8 \mathrm{meV}$ and $26.8 \mathrm{meV}$ respectively [3] [23]. 


\subsection{Specific Heat Capacity}

The specific heat capacity of a system is given by

$$
C_{V}=\frac{\partial E_{T}}{\partial T}
$$

For the three electron system, we substitute 10 into 9 so that,

$$
C_{V}=\left[\frac{E_{k}^{2}}{K_{B} T^{2}}\right] \mathrm{e}^{\left(\frac{-E_{k}}{K_{B} T}\right)}
$$

\subsection{Sommerfeld's Coefficient}

The electronic specific heat capacity $(\gamma)$ is determined from the specific heat capacity using the formula

$$
\gamma=\frac{C_{v}}{T}
$$

Putting (9) into (12) we get

$$
\gamma=\left[\frac{E_{k}^{2}}{K_{B} T^{3}}\right] \mathrm{e}^{\left(\frac{-E_{k}}{K_{B} T}\right)}
$$

\subsection{Entropy}

From the first law of thermodynamics temperature is defined as

$$
\frac{1}{T}=\frac{\mathrm{d} s}{\mathrm{~d} Q}
$$

From which we have,

$$
\mathrm{d} s=\frac{\mathrm{d} Q}{T}
$$

But recalling that $\mathrm{d} Q=C_{v} \mathrm{~d} T$ and integrating Equation (13), we have

$$
S=\int \frac{C_{v}}{T} \mathrm{~d} T
$$

Putting Equation (11) into Equation (14) to substitute for $C_{V}$, we have

$$
S=\int\left[\frac{E_{k}^{2}}{K_{B} T^{3}}\right] \mathrm{e}^{\left(\frac{-E_{k}}{K_{B} T}\right)} \mathrm{d} T
$$

So that the entropy becomes

$$
S=\int-K_{B}\left\{\left(\frac{E_{k}}{K_{B} T}-1\right) \mathrm{e}^{\frac{E_{k}}{K_{B} T}}\right\}
$$

\section{Results and Discussions}

\subsection{Energy of the System}

The total energy of a system results from the interaction between the particles of the system. The energy due to interaction between these particles increases with the temperature of the system. At $T_{O}$ point the material changes from a super- 
conducting to a normal state. Figure 1 shows the total energy of YBCO123 as a function of temperature.

This half-stretched sigmoid curve has been obtained previously by other researchers when they were relating energy of the system to temperature [24] [25] [26] [27]. From the graph, it is observed that the energy of the system increases with temperature but not linearly. At $T=0$, the electrons are completely non-interacting and thus the energy due to interaction between electrons interaction, $E_{T}=0$. The rise in temperature of the system beyond $18.5 \mathrm{~K}$ causes a significant change in the energy of interaction within the system. This phenomenon is shown in Figure 2.

As the temperature rises from $0 \mathrm{~K}$ to $18.5 \mathrm{~K}$, the Cooper pair formation is largely due to the singlet s-state because most of the electrons are in the ground state. The energy of a mole of YBCO123 within this temperature range is kept close to $0 \mathrm{~K}$ i.e. below $0.0007482 \mathrm{meV}$. When the temperature rises above $18.5 \mathrm{~K}$, some electrons enter the first excitation state where Cooper pair start forming in the singlet $\mathrm{p}$-state. The result is that the energy of the system starts to increase rapidly from below $0.0007482 \mathrm{meV}$. As the temperature increases towards the critical temperature, more electrons enter the first excitation state and more interactions between electrons in the singlet p-state increase leading to continued increase in the energy of the system. At $T=T_{C}$, the energy per unit mass for an

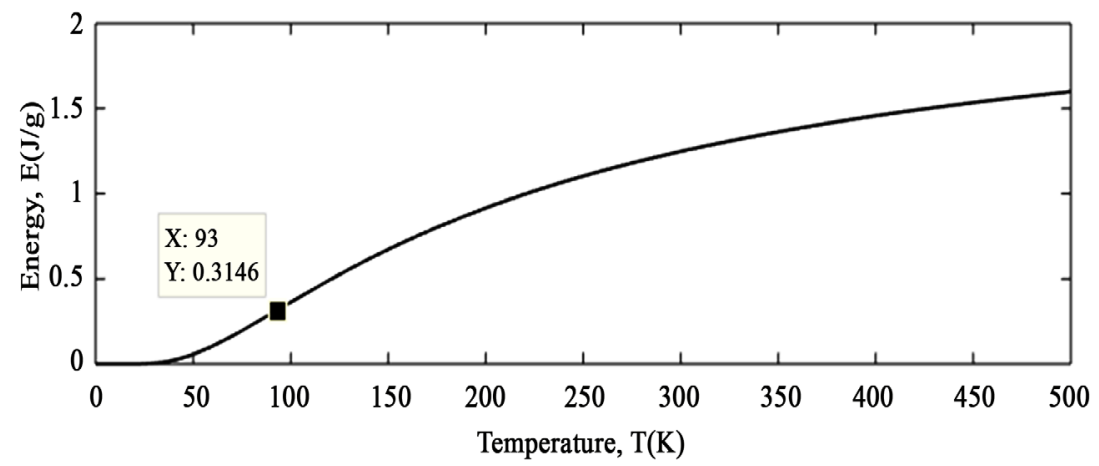

(a)

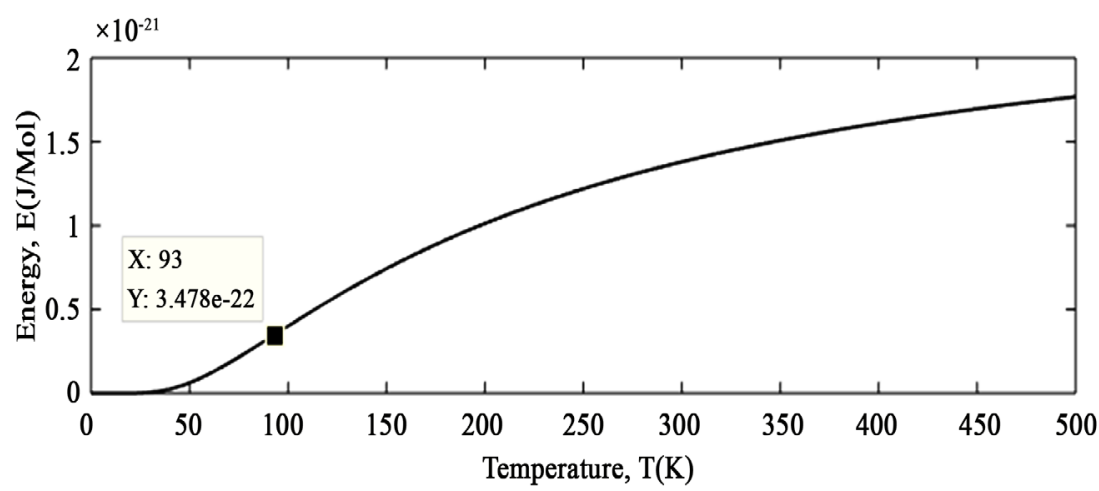

(b)

Figure 1. (a) Energy of a unit mass of Y123 as a function of temperature; (b) Energy of a mole of Y123 as a function of temperature. 


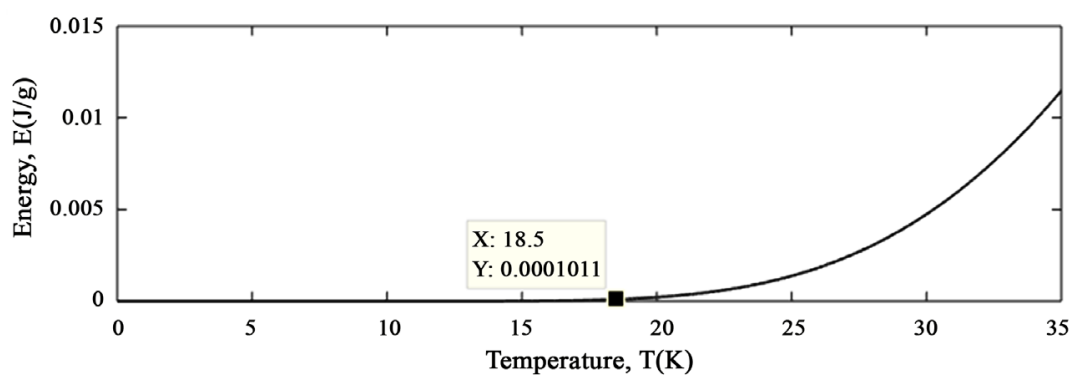

(a)

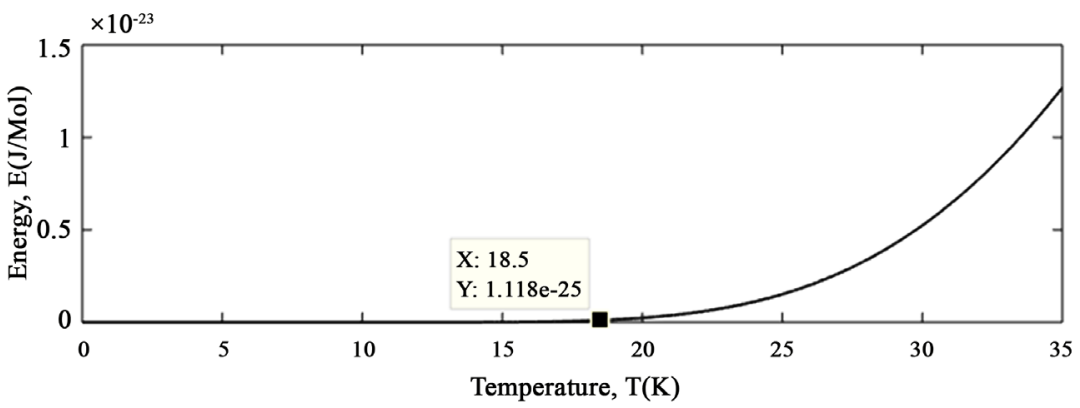

(b)

Figure 2. Energy in (a) $\mathrm{J}^{-\mathrm{g}^{-1}}$ and (b) $\mathrm{J} \cdot(\mathrm{Mol})^{-1}$ of the system as a function of temperature 0 $\mathrm{K}-35 \mathrm{~K}$.

electron interacting with a Cooper pair in Y123 is $314.7 \mathrm{~mJ} \cdot \mathrm{g}^{-1}$. This energy falls within the range for cuprates $\left(240 \mathrm{~J} \cdot \mathrm{kg}^{-1}-375 \mathrm{~J} \cdot \mathrm{kg}^{-1}\right)$ at $T_{C}$ as provided by Odhiambo (2016). The energy of a mole of YBCO123 at $T_{C}$ is $2.173 \mathrm{meV}$. This energy was determined at $T_{C}$ using second quantization method as $2.168 \mathrm{meV}$ [3].

The measured bulk energy for YBCO123 is $34 \mathrm{meV}$ [22]. The bulk energy represents the energy of interaction between the particles within the superconducting material [3]. Other than electron-Cooper pair interactions, there are other interactions within the structure of YBCO123 that contribute to the bulk energy. Thus, the energy of interaction between an electron and a Cooper pair is just a fraction of the measured bulk energy.

\subsection{Specific Heat Capacity}

Specific heat capacity of a substance is the amount of energy required to change the temperature of a unit mass of substance by $1 \mathrm{~K}$. The unit of specific heat is $\mathrm{J} \cdot \mathrm{kg}^{-1} \cdot \mathrm{K}^{-1}$. The specific heat of a substance depends on the temperature of the substance and is highest at the critical temperature. Figure 3 shows the specific heat as a function of temperature.

This shape concurs with those observed in previous research work [25] [27] [28]. From Figure 3, the specific heat capacity of YBCO123 increases with temperature of the system and attains its maximum at

$$
T=T_{C}
$$

where $C_{V}=6.757 \mathrm{~mJ} \cdot \mathrm{g}^{-1} \cdot \mathrm{K}^{-1}\left(4.669 \mathrm{~J} \cdot \mathrm{mol}^{-1} \cdot \mathrm{K}^{-1}\right)$. Odhiambo (2016) gives the 


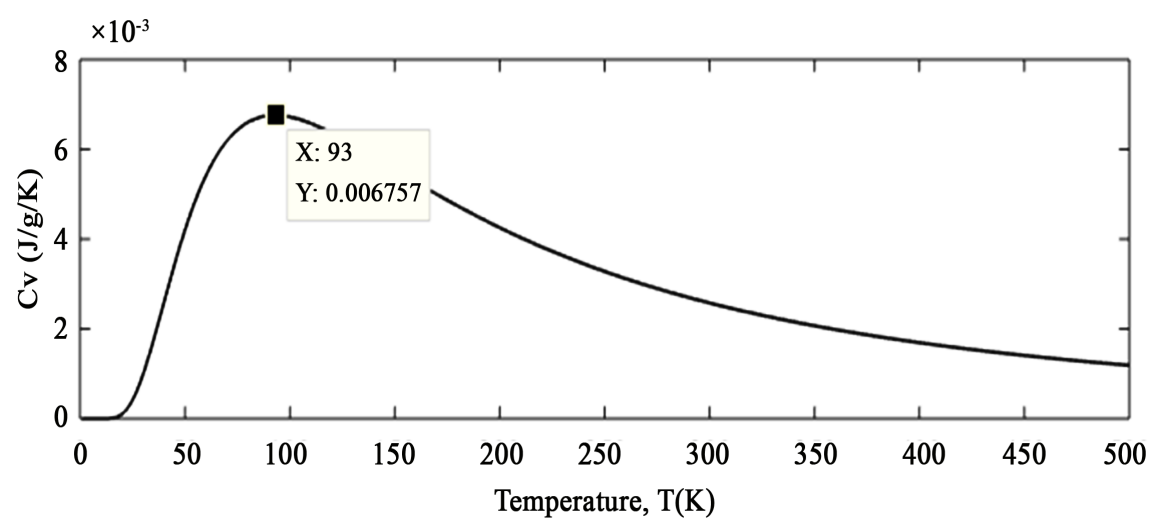

Figure 3. Specific heat capacity of a mole of YBCO123 as a function of $T / T_{C}$ and (b) Specific heat capacity of a mole of Y123 as a function of $T / \mathrm{T}_{\mathrm{C}}$.

specific heat of YBCO123 as $\left(4.526 \mathrm{~J} \cdot \mathrm{mol}^{-1} \cdot \mathrm{K}^{-1}\right)$. A theoretical study the pairing symmetry of the singlet and triplet pairing reveals that specific heat capacity of a $3 \mathrm{He}-4 \mathrm{He}$ mixture at $T_{C}$ is $28.91 \mathrm{~J} \cdot \mathrm{mol}^{-1} \cdot \mathrm{K}^{-1}$ [29]. Experimentally, the specific heat for YBCO123 at $T_{C}=91.68 \mathrm{~K}$ is $127.8 \mathrm{~J} \cdot \mathrm{mol}^{-1} \cdot \mathrm{K}^{-1}$ and the electronic contribution is about $1 \%-2 \%$ of the phonon contribution to the total specific heat [19] [30]. Elsewhere, the contribution electrons to the total specific heat has been given as $3.86 \%$ [31]. Below $T_{O}$ the specific heat dependence on temperature follows a power law indicating that the energy gap at Fermi surface has nodes in certain directions implying anisotropy in the energy gap [32]. This kind of energy gap suggests a d-wave pairing in the energy gap.

\subsection{Sommerfeld's Coefficient}

The Sommerfeld's coefficient of a material relates the heat capacity of the material to a specific temperature. Figure 4 shows a graph of specific heat capacity of YBCO123 as a function of (a) temperature (b) $T / T_{C}$

Using the graph, the Sommerfeld's coefficient increases with temperature and is optimum at $T / T_{C}=0.6666$ where $\gamma=0.09031 \mathrm{~mJ} \cdot \mathrm{g}^{-1} \cdot \mathrm{K}^{-2}$, which upon conversion becomes $62.4 \mathrm{~mJ} \cdot \mathrm{mol}^{-1} \cdot \mathrm{K}^{-2}$. This value is again close to $60.4 \mathrm{~mJ} \cdot \mathrm{mol}^{-1} \cdot \mathrm{K}^{-2}$ that was obtained by second quantization method [3]. On the other hand, a high resolution differential technique has given this value as $60 \mathrm{~mJ} \cdot \mathrm{mol}^{-1} \cdot \mathrm{K}^{-2}[19]$.

\subsection{Entropy}

Entropy of a system is the measure of disorder within the system. Increase in temperature of the system increases the agitation of the particles hence increase in entropy. When the entropy is very high, the enhanced disorder hence increased energy within the system destroys Cooper pairs. Consequently, the material changes from being superconducting state to a normal state or vice versa. The lower the entropy of a system, the higher the orderliness of its particles. Figure 5 shows the relationship between entropy of YBCO123 and its temperature. 


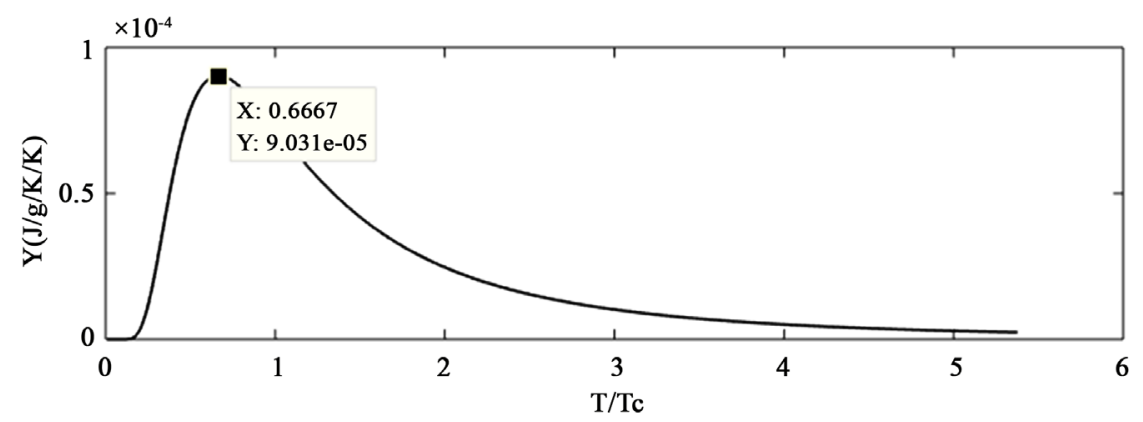

Figure 4. Sommerfeld's coefficient per unit mass of YBCO123 as a function of $T / T_{C}$

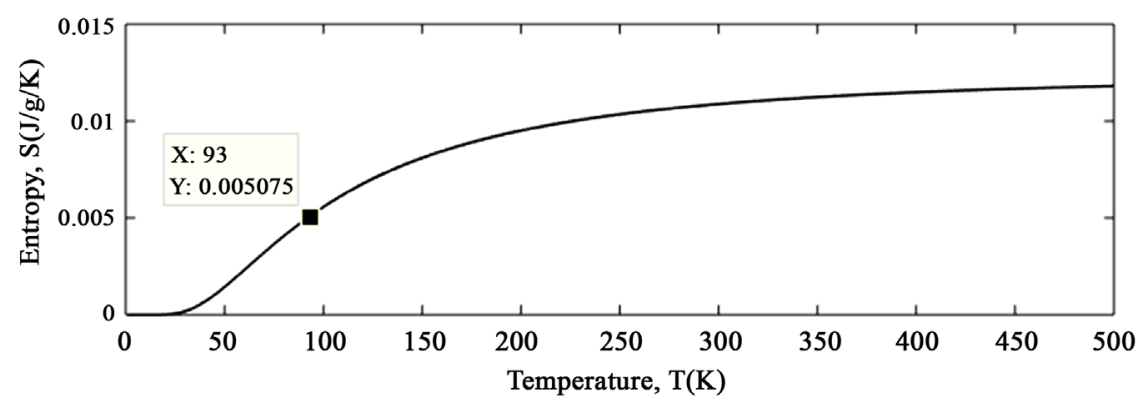

Figure 5. Entropy of a unit mass of Y123 as a function of temperature.

The entropy of the system decreases with temperature implying increase in the orderliness of the system with decrease in temperature. At lower temperature, the entropy is zero to imply that the system is highly ordered. Above this entropy, the increased energy of the system agitates the lattice particles and the superconductivity of the system is destroyed. The lower the entropy of a system, the higher the order of its particles. At $T=0$, the entropy of the system is zero and the particles within the system, at this point, are highly ordered. Figure 6 shows the change in the entropy as the temperature rises from $0 \mathrm{~K}$ to $35 \mathrm{~K}$.

Within the temperature range $0 \leq T \leq T_{C}$, the entropy YBCO123 is maintained below $0.0002083 \mathrm{meV} \cdot \mathrm{mol}^{-1} \cdot \mathrm{K}^{-1}$. When the temperature rises above $17.5 \mathrm{~K}$, some electrons are excited into the first excitation state where electrons pair in the singlet p-state. At this point, disorder starts setting in. As the temperature increases up to the critical temperature, more electrons enter the first excitation state and more electrons pair up in the singlet p-state leading to continued decrease in the order of the system. Therefore, a singlet s-state is more ordered than the singlet p-state.

Similar curves were obtained by other authors [18] [20] [24]. Figure 7 shows entropy as a function of $T / T_{C}$

Figure 7 shows that at $T=T_{C}=93 \mathrm{~K}$ that the entropy $S=5.075 \mathrm{~mJ} \cdot \mathrm{K}^{-1}$. Upon conversion, the entropy becomes $3.507 \mathrm{meV} \cdot \mathrm{K}^{-1}$. The interaction of singlet and triplet state in a heavy fermion superconductor gives an entropy of $3.5 \times$ $10^{-21} \mathrm{~J} \cdot \mathrm{K}^{-1}[29]$ while dipole mediated $\mathrm{t}-\mathrm{J}$ model $(\mathrm{t}-\mathrm{J}-\mathrm{d})$ gives a maximum entropy of $\left(5.04694 \times 10^{-22} \mathrm{~J} \cdot \mathrm{K}^{-1}\right)$ [20]. Loram et al., (1993), experimentally determined entropy to range between $0.06-0.22 K_{B}$ (Unit cell) ${ }^{-1}$ when holes were varied 


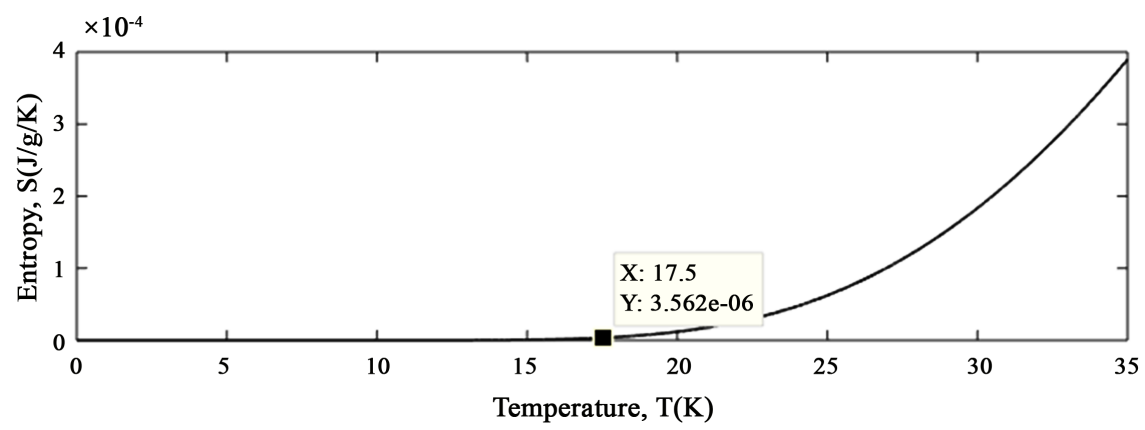

(a)

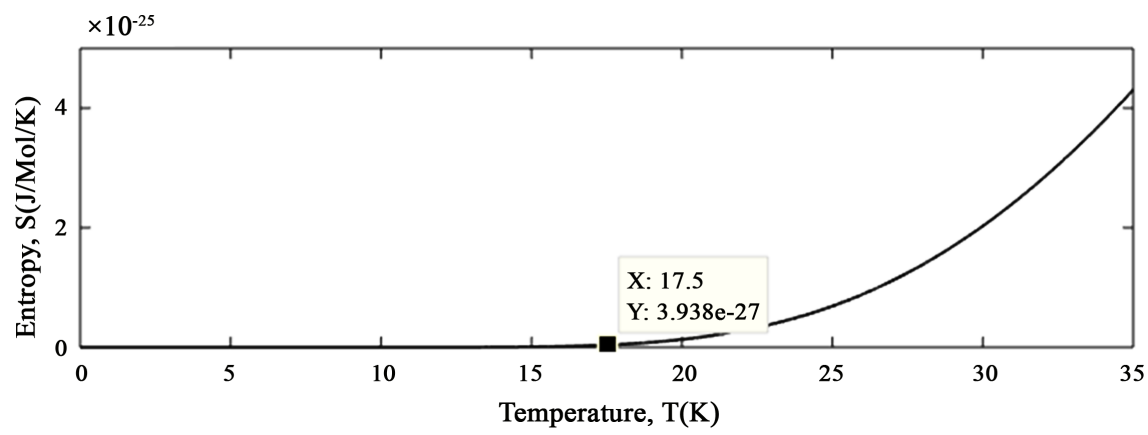

(b)

Figure 6. The entropy (a) $\mathrm{J} \cdot \mathrm{g}^{-1} \cdot \mathrm{K}^{-1}$; (b) $\mathrm{J} \cdot(\mathrm{Mol})^{-1} \cdot \mathrm{K}^{-1}$ of the system between $0 \mathrm{~K}$ to $35 \mathrm{~K}$.

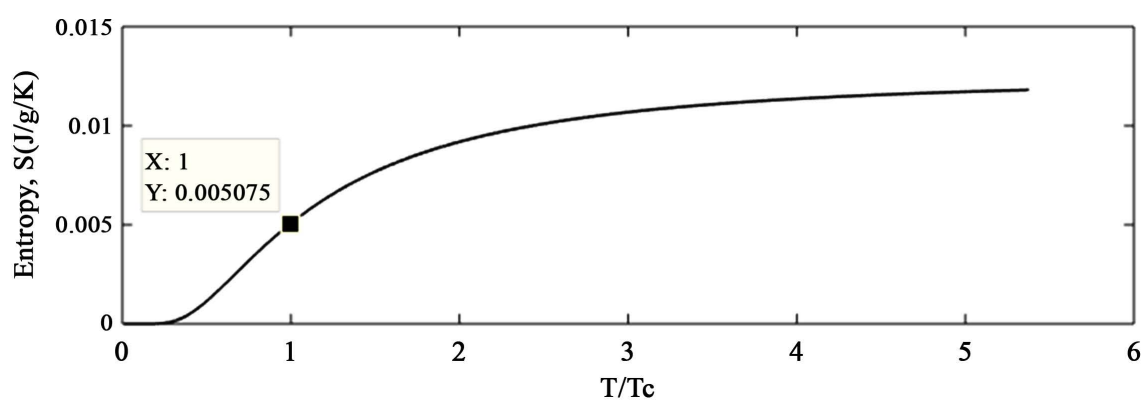

(a)

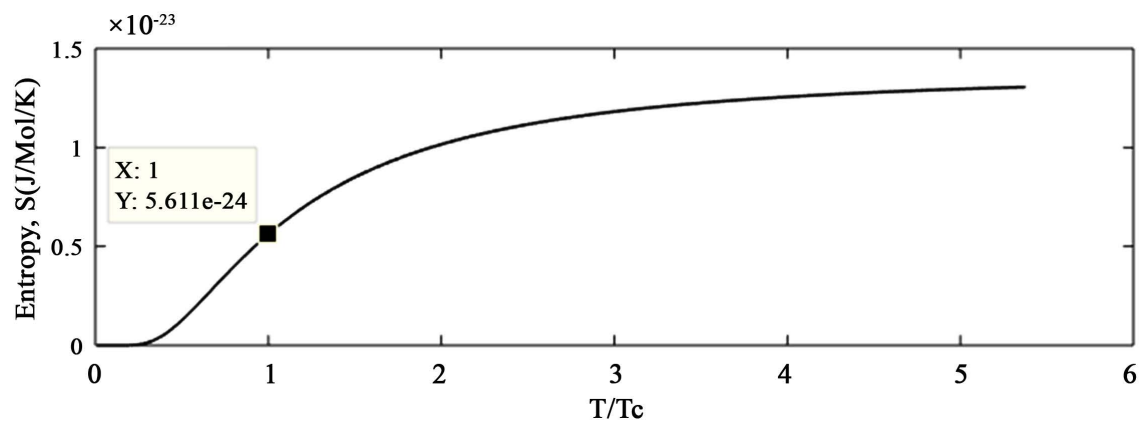

(b)

Figure 7. (a) Entropy per unit mass of YBCO123 as a function of $T / T_{C}$ (b) Entropy of amole of YBCO123 as a function of $T / T_{C}$.

from $0.57-0.97$ (Unit cell) $)^{-1}$ [19]. This is an equivalent of $8.28 \times 10^{-25}-3.036 \times$ $10^{-24} \mathrm{~J} \cdot(\text { Unit cell) })^{-1} \cdot \mathrm{K}^{-1}$. When the temperature of a material rises above its $T_{C}$ 
the increased energy of the system agitates the lattice vibrations (increased disorder), leading to a higher entropy. In that condition, no Cooper pair exists and thus superconductivity of the material is destroyed. However, below this entropy, the system exhibits orderliness resulting into the formation Cooper pairs. Figure 8 shows the change in the entropy as the temperature rises from $0 \mathrm{~K}$ to $35 \mathrm{~K}$.

Within this range of temperature, the entropy YBCO123 is below 0.0002083 $\mathrm{meV} \cdot \mathrm{mol}^{-1} \cdot \mathrm{K}^{-1}$. When the temperature rises above $17.5 \mathrm{~K}$, some electrons gain access to the first excitation state where electrons pair in the singlet $\mathrm{p}$-state. At this point, disorder sets in slowly. As the temperature increases up to the critical temperature, more electrons enter the first excitation state and more electrons pair up in the singlet p-state leading to a less ordered system. Therefore, a singlet $\mathrm{s}$-state is more ordered than the singlet p-state.

\section{Conclusions}

We note that the energy of interaction between a Cooper pair and an electron increases with temperature in the range $18 \leq T \leq T_{C}$. The energy of interaction within $\mathrm{Y} 123$ at $T=T_{C}$, as determined in this study, is $2.174 \mathrm{meV} \cdot \mathrm{mol}^{-1}$. As the energy of the system increases from zero as the Cooper pairing changes from singlet s-state to singlet p-state. The energy of interaction between the electron and the Cooper pair determined in this study accounts for $6.39 \%$ of the total energy.

The specific heat of YBCO123 increases with temperature with the peak at

$$
T=T_{C}
$$

where $C_{V}=4.669 \mathrm{~J} \cdot \mathrm{mol}^{-1} \cdot \mathrm{K}^{-1}$. In the region $T>T_{C}$, the specific heat decreases with increase in the temperature of the system. In the range $18 \leq T \leq T_{C}$, the Sommerfeld's coefficient increases with temperature and is highest at

$$
T=\frac{2}{3} T_{C}
$$

where $\gamma=0.09036 \mathrm{~mJ} \cdot \mathrm{g}^{-1} \cdot \mathrm{K}^{-2}$, which upon conversion becomes 62.4 $\mathrm{mJ} \cdot \mathrm{mol}^{-1} \cdot \mathrm{K}^{-2}$. The entropy of YBCO123 at $T / T_{C}=1$ was found to be $3.84 \mathrm{meV} \cdot \mathrm{K}^{-1}$. The electron pairing is more ordered in singlet s-state (below $18 \mathrm{~K}$ ) than in the singlet $\mathrm{p}$-state (above $18 \mathrm{~K}$ ).

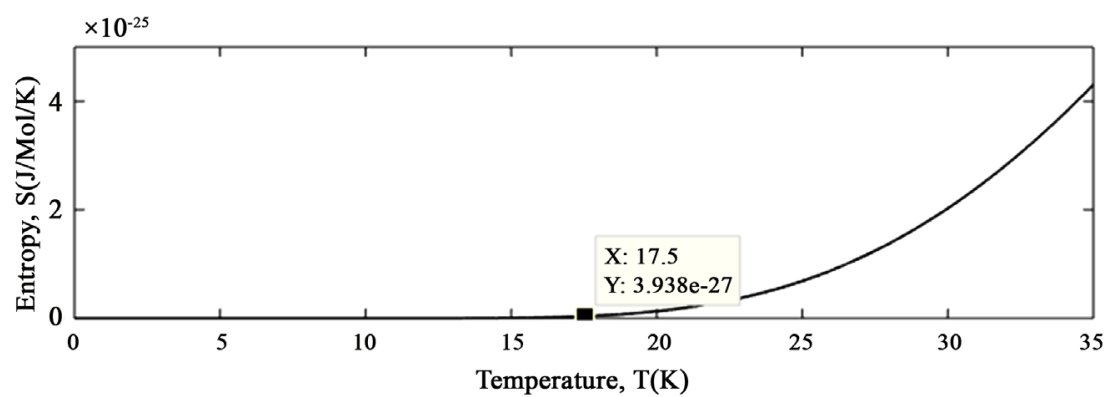

Figure 8. The entropy of the system between $0 \mathrm{~K}$ to $17.5 \mathrm{~K}$. 
This study has shown that the energy, specific heat, Sommerfeld's coefficient and entropy of YBCO123 remain almost constant (and close to zero) as temperature rises from zero to about $18 \mathrm{~K}$ due to electron pairing in the singlet-s state. Above $T=18 \mathrm{~K}$ the energy of $\mathrm{YBCO} 123$ rises sharply owing to the electron pairing in singlet-p state. In this region, high energy of up to $2.174 \mathrm{meV}$ per Mole is involved. The change in electron pairing from a purely singlet-s environment to a combination of both singlet-s and singlet-p states might be an avenue to understanding high temperature superconductivity, owing to the variation of energy with temperature associated with the change in the pairing environment.

\section{Conflicts of Interest}

The authors declare no conflicts of interest regarding the publication of this paper.

\section{References}

[1] Bardeen, J., Cooper, L.N. and Schrieffer, J.R. (1957) The Microscopic Theory of Superconductivity. Physics Review, 108, Article ID: 1175. https://doi.org/10.1103/PhysRev.108.1175

[2] Wollman, D.A., Van Harlingen, D.J., Lee, W.C., Ginsberg, D.M. and Leggett, A.J. (1993) Experimental Determination of Superconducting Pairing State of YBCO from the Phace Coherence of YBCO-Pb dc SQUIDs. Physical Review Letter, 71, Article ID: 2134. https://doi.org/10.1103/PhysRevLett.71.2134

[3] Odhiambo, J.P.O. (2016) Thermodynamic Properties of High Temperature Superconducting Cuprates Due to Interactions between Cooper Pairs and Electrons. Ph.D. (Physics) Thesis, MasindeMuliro University of Science and Technology, Kakamega.

[4] Tsuei, C. and Kirtley, R., (2002) d-wave Pairing Symmetry in Cuprate Superconductors-Fundamental Implications and Potential Applications. Physica C: Superconductivity, 367, 1-8. https://doi.org/10.1016/S0921-4534(01)00976-5

[5] Bednorz, G.J. and Muller, K.A. (1986) Possible High $T_{C}$ Superconductivity in the La-Ba-Cu-O System. Zeitschrift für Physik B Condensed Matter, 64, 189-193.

[6] Wu, M.K., Ashburn, J.R., Torng, C.J., Hor, P.H., Meng, R.L., Gao, L., Huang, Z.J., Wang, Y.Q. and Chu, C.W. (1987) Superconductivity at $93 \mathrm{~K}$ in a New Mixed-Phase Y-Ba-Cu-O Compound System at Ambient Pressure. Physical Review Letters, 58, Article ID: 908. https://doi.org/10.1103/PhysRevLett.58.908

[7] Maeda, H., Tanaka, Y., Fukutumi, M. and Asano, T. (1988) A New High $T_{C}$ Oxide Superconductor without a Rare Earth Element. Japanese Journal of Applied Physics, 27, L209-L210.

[8] Schilling, A., Cantoni, M., Guo, J.D. and Ott, H.R. (1993) Superconductivity in Hg-Ba-Ca-Cu-O System. Nature, 363, 56-58.

https://doi.org/10.1038/363056a0

[9] Ihara, H., Hirobayashi, M., Tanino, H., Tokiwa, K., Ozawa, H., Akahana, Y. and Kawamura, $\mathrm{H}$. (1993) The Resistivity Measurements of $\mathrm{HgBa}_{2} \mathrm{Ca}_{2} \mathrm{Cu}_{3} \mathrm{O}_{8+X}$ and $\mathrm{HgBa}_{2} \mathrm{Ca}_{3} \mathrm{Cu}_{4} \mathrm{O}_{10+x}$ Superconductors under High Pressure. Japanese Journal of Applied Physics, 32, L1732-L1734. https://doi.org/10.1143/JJAP.32.L1732 
[10] Onbasli, U., Wang, Y.T., Naziripour, A., Tello, R., Kiehl, W. and Hermann, A.M. (1996) Transport Properties of High- $T_{C}$ Mercury Cuprates. Physica Status Solidi (b), 194, 371-382. https://doi.org/10.1002/pssb.2221940131

[11] Drozdov, A.P., Eremets, M.I., Troyan, I.A., Ksenofontov, V. and Shylin, S.I. (2015) Conventional Superconductivity at 203 Kelvin at High Pressures in the Sulfur Hydride System. Nature, 525, 73-76. https://doi.org/10.1038/nature14964

[12] Cilento, F., Conte, D., Coslovich, D., Peli, S., Nembrini, N., Mor, S., Banfi, F., Ferrini, G., Eisaki, H., Chan, M.K., Dorow, C.J., Veit, M.J., Greven, M., Marel, D., Comin, R., Damscelli, A., Retig, L., Bovoenspien, U., Capona, M., Gianetti, C. and Parmigiani, F. (2014) Photo-Enhanced Antinodal Conductivity in the Pseudogap State of $T_{C}$ Cuprates. Nature Communication, 5, Article No. 4353. https://doi.org/10.1038/ncomms5353

[13] Howald, L., Stilp, E., de Reotier, D.P., Yaouanc, A., Raymond, S., Piamonteze, C., Lapertot, G., Baines, C. and Keller, H. (2015) Evidence of Coexistence of Bulk Superconductivity and Itinerant Antiferromagnetism in the Heavy Fermion System CeCo $\left(\operatorname{In}_{1-x} \mathrm{Cd}_{x}\right)_{5}$. Scientific Report, 5, Article No. 12528. https://doi.org/10.1038/srep12528

[14] Odhiambo, J.O., Sakwa, T.W., Ayodo, Y.K. and Rapando, B.W. (2016) Thermodynamic Properties of Mercury Based Cuprate Due to Cooper Pair-Electron Interaction. Journal of Multidisciplinary Engineering Science and Technology, 3, 5241-5248.

[15] Chubukov, A.V., Efremov, D.V. and Eremin, I. (2008) Magnetism, Superconductivity and Pairing Symmetry in Iron-Based Superconductors. Physics Review B, 78, Article ID: 134512. https://doi.org/10.1103/PhysRevB.78.134512

[16] Malik, M.A. and Malik, B.A. (2014) High Temperature Superconductivity: Materials, Mechanisms and Applications. Bulgarian Journal of Physics, 41, 305-314.

[17] Dahm, T., Hinkov, V., Borisenko, V., Kordyuk, A.A., Zabolotny, V.B., Fink, J., Buchner, B., Scalapino, D.J., Hanke, W. and Keimer, B. (2009) Strength of the Spin-Fluctuation-Mediated Pairing Interaction in High-Temperature Superconductor. Nature Physics, 5, 217-221. https://doi.org/10.1038/nphys1180

[18] Kibe, H.E. (2015) Themodynamic Properties of Heavy Fermion Superconductors. M.Sc. (Physics) Thesis, MasindeMuliro University of Science and Technology, Kakamega.

[19] Loram, J.W., Mirza, K.A., Cooper, J.R. and Liang, W.Y. (1993) Electronic Specific Heat of $\mathrm{Yba}_{2} \mathrm{Ca}_{3} \mathrm{Cu}_{3} \mathrm{O}_{6+\mathrm{x}}$ from 1.8 to 300K. Physics Review Letter, 71, 1740. https://doi.org/10.1103/PhysRevLett.71.1740

[20] Rapando, B.W., Khanna, K.M., Tonui, J.K., Sakwa, T.W., Muguro, K.M., Kibe, H., Ayodo, Y.K. and Sarai, A. (2015) The Dipole Mediated t-J Model for High-T $\mathrm{C}_{\mathrm{Su}}$ perconductivity. International Journal of Physics and Mathematical Sciences, 5, $32-37$.

[21] Edwards, H.L., Markert, J.T. and de Lozanne, A.L. (1992) Energy Gap and Surface Structure of $\mathrm{YBa}_{2} \mathrm{Cu}_{3} \mathrm{O}_{7-\mathrm{x}}$ Probed by Scanning Tunneling Microscopy. Physics Review Letters, 69, 2967. https://doi.org/10.1103/PhysRevLett.69.2967

[22] Nakayama, K., Sato, T., Terashima, K., Matsu, H., Takahash, T., Kubota, M., Ono, K., Nishizaki, T., Takahashi, Y. and Kobayashi, N. (2007) Bulk and Surface Low-Energy Excitations in $\mathrm{YBa}_{2} \mathrm{Cu}_{3} \mathrm{O}_{7-\delta}$ Studied by High-Resolution Angle-Resolved Photoemission Spectroscopy. Physical Review B, 75, Article ID: 014513. https://doi.org/10.1103/PhysRevB.75.014513

[23] Mazur, D., Zasadzinski, J.F., Gray, K.E., Ma, Y.C., Wang, N.L. and Yan, S.L. (2006) 
Tunnelling Spectroscopy of $\mathrm{Tl}_{2} \mathrm{Ba}_{2} \mathrm{CaCu}_{2} \mathrm{O}_{8}$ Single Crystal and Thin Film. http://arxiv.org/abs/cond-mat/0611636v1

[24] Odhiambo, J.O. and Makokha, J.W. (2018) Specific Heat and Entropy of a Three-Electron Model in Bismuth Based Cuprate Superconductor. World Journal of Applied Physics, 3, 19-24.

[25] Kibe, H.E., Sakwa, T.W. and Khanna, K.M. (2017) Specific Heat of the Integrated S-Wave and P-Wave Pairing in Uranium and Cerium Based Heavy Fermion Superconductors. International Journal of Physics and Mathematical Sciences, 7, 1-6.

[26] Ketterson, B. and Song, S.N. (1999) Superconductivity. Cambridge University Press, Cambridge. https://doi.org/10.1017/CBO9781139171090

[27] Mumali, O.W., Ayodo, Y.K., Ndinya, B.O. and Odhiambo, J.O. (2016) Quantum Thermodynamics of a Strongly Interacting Bose-Fermi Mixture in a Three-Dimensional Anharmonic Potential. International Journal of Physics and Mathematical Sciences, 6, 41-50.

[28] Sereni, J.G., Curlik, I., Giovannini, M., Strydom, A. and Reiffers, M. (2018) Physical Properties of the Very Heavy Fermion $\mathrm{YbCu}_{4} \mathrm{Ni}$. Physical Review B, 98, Article ID: 094420. https://doi.org/10.1103/PhysRevB.98.094420

[29] Bessergeven, V.G., Kovalevskaya, Y.A., Naymov, V.N. and Frolova, G.I. (1995) Phonon Characteristics of $\mathrm{YBa}_{2} \mathrm{Cu}_{3} \mathrm{O}_{7-\delta}$. Physica C, 245, 36-40. https://doi.org/10.1016/0921-4534(95)00096-8

[30] Shaviv, R., Westrum, E.F., Brown, R.J.C., Sayer, M., Yu, X. and Weir, R.D. (1990) The Heat Capacity and the Derived Thermophysical Properties of the High $\mathrm{T}_{\mathrm{C}} \mathrm{Su}$ perconductors $\mathrm{YBa}_{2} \mathrm{Cu}_{3} \mathrm{O}_{8-\delta}$ from 5.3 to $350 \mathrm{~K}$. Journal of Chemical Physics, 92, 6794-6799. https://doi.org/10.1063/1.458266

[31] Andrei, M. (2004) Room Temperature Superconductivity. Cambridge International Sciences Publishing, Cambridge.

[32] Kibe, H.E., Sakwa, T.W., Ayodo, Y.K., Rapando, B.W., Khanna, K.M. and Sarai, A. (2015) Thermodynamic Properties of Heavy Fermion Superconductors. International Journal of Physics and Mathematical Sciences, 5, 23-33. 